\title{
Indoor Distributed Antenna Systems for Multi-Storey Buildings
}

\author{
Temitope Alade* and Jiangzhou Wang ${ }^{\dagger}$ \\ ${ }^{*}$ University of Worcester, ${ }^{\dagger}$ University of Kent, Canterbury, United Kingdom
}

\begin{abstract}
One of the main targets of the fifth generation (5G) mobile communication systems and beyond is providing high data rate wireless transmissions and ubiquitous coverage to users. To support increasing subscriber populations in indoor environments, indoor wireless communication systems are required, but frequency spectrum allocations are limited and requires reuse of the limited spectrum. In order to design efficient and reliable indoor communication systems, a thorough understanding of the reuse distance is vital. This paper investigates a number of important issues associated with indoor radio propagation and frequency reuse planning in multi-storey buildings employing indoor distributed antenna system (DAS), where distributed remote antenna units (RAUs) supported by a central unit $(\mathrm{CU})$ communicate with mobile equipment (MEs). In the DAS, co-channel interference caused by frequency reuse is a restraining factor as the frequency reuse distances are generally smaller. The effect of different reuse distances, penetration losses, pathloss exponents, and co-channel interference on spectral efficiency is analysed and performance evaluated over a wide range of potential ME locations.

Index Terms-Distributed antenna system (DAS), Co-channel interference, Multi-floor in-building propagation, Spectral efficiency.
\end{abstract}

\section{INTRODUCTION}

To address the explosive growth in wireless data traffic, the wireless industry is moving to its fifth generation $(5 \mathrm{G})$ of mobile communication systems that will offer data rates in the range of Gigabits-per-second (Gbps), ultralow latency, improved coverage and reliability, and enable billions of connected devices (Internet of Things) [1]. However, delivering ubiquitous and high quality wireless services to users in indoor regions such as high-rise buildings remains a major challenge. This is essentially due to long radio transmission distance, limited transmit power, limited bandwidth and electromagnetic shielding of wireless signals by walls inside buildings [2]. Moreover, penetration losses are more prominent at higher frequencies likely to be used for future mobile communications [3].

Several techniques such as massive multiple-inputmultiple-output (MIMO), millimeter wave (mmWave) networks, non-orthogonal multiple access (NOMA) and small cells are being considered for $5 \mathrm{G}$ to enhance data rates and improve wireless coverage [4]-[8]. To improve cellular system performance in multi-floor in-building environments, a promising additional solution is distributed antenna systems (DASs) [9], where distributed remote antenna units (RAUs) supported by a central unit (CU) communicate with mobile equipment (MEs). DASs have drawn considerable attention in recent years due to its enormous improvement in terms of better coverage, lower transmit power and higher data rates
[10]-[15]. With DASs, the radio transmission distance from an ME to the surrounding RAUs is reduced and a high signalto-noise ratio (SNR) can be achieved.

On the other hand, indoor wireless systems are particularly susceptible to co-channel interference due to frequency reuse between indoor cells [16]. Unlike two-dimensional planar frequency reuse strategies employed in outdoor DAS where the resulting co-channel interference from neighboring cells can be reduced with careful deployment techniques, reducing co-channel interference for indoor DAS is a challenge. This is mainly because the indoor propagation environment is considerably more compact, and all RAUs and MEs are located in close proximity. In order to achieve ubiquitous coverage of the service area, an operator would need many more cells. Therefore, knowledge of reuse distances within the building is important if efficient indoor DASs are to be designed. Furthermore, due to large variability in building layouts, architectural styles, and building materials [17], it is important to explore how radio-waves propagate within and around buildings, and give rise to co-channel interference in multi-floor indoor wireless systems.

This paper investigates the performance of indoor DAS in the presence of co-channel interference. Indoor radio channel propagation characteristics in multi-storey buildings, with frequency reuse among floors, and the resulting impact on system performance are examined.

\section{SYSTEM MODEL}

Fig. 1 shows the uplink of an indoor DAS in a multi-storey building where each storey in the building is treated as a cell with the floors forming natural boundaries. RAUs are evenly located across every floor to serve all MEs on each floor. The RAUs are connected to the CU via high bandwidth links such as optical fibre or cable. Each floor of the building has a similar construction with an internal wall, and frequency reuse is employed among the floors.

In the building considered, there are a total of $K$ CUs, one on each floor and each $\mathrm{CU}$ is equipped with $N$ RAUs. Each floor has a common inter-floor spacing of $F$ meters, and one active ME equipped with a single antenna is evenly located across each floor at height of $v$ meters. The reuse distance $C$ measured in floors, is the distance at which the frequency resource can be reused (Fig. 1 illustrates a reuse distance of three floors). Thus, $F C$ is the total reuse distance in meters. In dense urban environments, there are likely to be nearby buildings, accordingly, it is assumed that there is a multi-storey building located a few meters away from the 
reference building. Analysis of both line-of-sight (LOS) and non-LOS (NLOS) propagation conditions are considered.

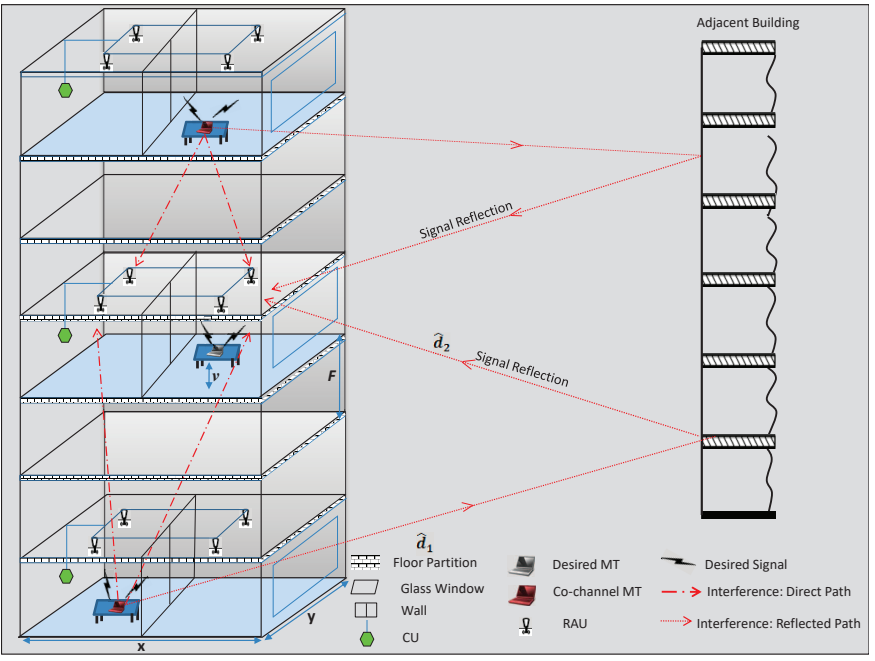

Fig. 1. Indoor DAS with frequency reuse distance of three floors

\section{A. Propagation mechanisms in multi-storey buildings}

Measurement campaigns in multi-storey buildings have been conducted by several studies [18]-[20]. The general trend suggests that signal losses are determined by floor plans, construction materials used, peoples' positions and movements, type and number of office equipment, scale of MEs used, and so on. Where there is an adjacent building, there are indications that reflections from the building contribute to propagation between floors of the victim building [21]. Accordingly, this paper assumes that interfering signals from other MEs arrive from co-channel floors via inter-floor penetration and reflections from the adjacent building.

\section{B. Channel model}

The model of the channel between ME- $k$ and $n$-th RAU on the reference floor is the sum of low pass equivalent impulse responses of the individual paths expressed as

$$
H_{k, n}(t)=h_{k, n}^{\prime}(t)+\hat{h}_{k, n}(t)
$$

where $h_{k, n}^{\prime}(t)$ and $\hat{h}_{k, n}(t)$ denote the impulse responses for inter-floor paths, and the reflected paths from the adjacent building, respectively. $h_{u, n}^{\prime}(t)$ is given by

$$
h_{k, n}^{\prime}(t)=\left(d_{k, n}\right)^{-\mu / 2} \cdot \tilde{\varphi}_{k, n}^{l / 2} \cdot \varphi_{u, n}^{r / 2} \cdot \alpha_{k, n} \cdot e^{j \theta_{k, n}} \cdot \delta\left(t-\tau_{k, n}\right)
$$

where $d_{u, n}$ is the distance between ME- $k$ and the $n$-th RAU on the reference floor. $\mu$ is the path loss exponent, typically between 2 to 6 depending on the propagation condition [21]. $\tilde{\varphi}_{k, n}$ and $\varphi_{k, n}$ are the penetration loss through a single wall and a single floor respectively, and $l$ and $r$ are the number of walls and floors in the transmission path respectively. $\alpha_{k, n}$, $\theta_{k, n}$ and $\tau_{k, n}$ are the channel fading coefficient, path phase, and path delay, respectively, and are statistically independent. It is assumed that $\theta_{k, n}$ and $\tau_{k, n}$ are uniformly distributed over $[0,2 \pi]$ and $\left[0, T_{s}\right]$, respectively. $\delta(t)$ denotes the Dirac delta function. Specifically, $\alpha_{k, n}$ undergoes Nakagami fading, hence, $\alpha_{k, n}^{2}$ is Gamma distributed with the probability distribution function (pdf) expressed as [22]

$$
\begin{gathered}
p_{\alpha_{k, n}^{2}}\left(\alpha_{k, n}^{2}\right)=\left(\frac{m_{n}}{\Omega_{n}}\right)^{m_{n}} \frac{\left(\alpha_{k, n}^{2}\right)^{m_{n}-1}}{\Gamma\left(m_{n}\right)} \exp \left(-\frac{m_{n}}{\Omega_{n}} \alpha_{k, n}^{2}\right), \\
\alpha_{k^{\prime}, n} \geq 0
\end{gathered}
$$

where $m_{n}$ is the Nakagami factor and $\Omega_{n}$ is the average fading power of the received signal. The parameters $\Omega_{n}$ and $m_{n}$ can be expressed as

$\Omega_{n}=E\left[\alpha_{k, n}^{2}\right]$ and $m_{n}=\frac{\Omega_{n}^{2}}{E\left[\left(\alpha_{k, n}^{2}-\Omega_{n}\right)^{2}\right]}, m_{n} \geq \frac{1}{2}$ respectively. When $m_{n}=1$, Nagakami fading reduces to Rayleigh fading [23]-[26]. $\hat{h}_{k, n}(t)$ in (1) is given by

$\hat{h}_{k, n}(t)=\left(\hat{d}_{k, n}\right)^{-\hat{\mu} / 2} \cdot \hat{\varphi}_{k, n}^{1 / 2} \cdot \ddot{\varphi}_{k, n} \cdot \tilde{\varphi}_{k, n}^{l / 2} \cdot \hat{\alpha}_{k, n} \cdot e^{j \hat{\theta}_{k, n}} \cdot \delta\left(t-\hat{\tau}_{k, n}\right)$

where $\hat{d}_{k, n}$ denotes the distance between ME- $k$ and the $n$ th RAU on the reference floor when the signal is reflected by the adjacent building as illustrated in Fig. $1 . \hat{\mu}$ is the path loss propagation exponent of the reflected path towards the adjacent building (free space propagation is assumed, $\hat{\mu}=2), \hat{\varphi}_{k, n}$ is the reflection coefficient at the building surface and $\ddot{\varphi}_{k, n}$ is the transmission loss through the glass windows. it is assumed that the reflected path experiences glass penetration loss twice. $\hat{\alpha}_{k, n}, \hat{\theta}_{k, n}$ and $\hat{\tau}_{k, n}$ are the channel fading coefficient, path phase and channel delay, respectively, for the reflected path. Due to no line of sight path, $\hat{\alpha}_{k, n}$ is assumed to be Rayleigh distributed with the mean square $E\left[\alpha_{u, n}^{2}\right]=1$. It is assumed that $\hat{\theta}_{k, n}$ and $\hat{\tau}_{k, n}$ have the same distribution as $\theta_{k, n}$ and $\tau_{k, n}$ respectively.

\section{Transmit and receive signal model}

The transmitted signal from ME- $k$ to the $n$-th RAU is given by

$$
x_{k}(t)=\operatorname{Re}\left[\sqrt{P_{s}} \sum_{i=-\infty}^{\infty} b_{k}[i] \rho_{T_{s}}\left(t-i T_{s}\right) e^{j 2 \pi f_{c} t}\right]
$$

where $R e[x]$ represents the real part of $x, P_{s}$ is the transmit power which is assumed to be the same for all MEs, $b_{k}[i]$ is the transmitted symbol, $T_{s}$ represent the symbol duration, $E\left[b_{k}\right]=0$ and $E\left[b_{k}\right]^{2}=1 . \rho_{T_{s}}(t)$ is a pulse waveform defined as $\rho_{T_{s}}(t)=1$ for $0 \leq t \leq T_{s}$ and $\rho_{T_{s}}(t)=0$ otherwise and $f_{c}$ is the carrier frequency.

Accordingly, the received signal by the $n$-th RAU can be represented by its complex low-pass equivalent as

$$
\begin{aligned}
r_{k, n}(t) & =D_{s} \cdot x_{k^{\prime}}\left(t-\tau_{k^{\prime}, n}\right) \\
& +\sum_{k=1, k \neq k^{\prime}}^{K}\left(G_{d}+Q_{r}\right) \cdot x_{k}\left(t-\tau_{k, n}\right)+\eta_{n}(t)
\end{aligned}
$$

where $D_{s}=\left(d_{k^{\prime}, n}\right)^{-\lambda / 2} \cdot \tilde{\varphi}_{k^{\prime}, n}^{l / 2} \cdot \alpha_{k^{\prime}, n} \cdot e^{j \theta_{k^{\prime}, n}}, G_{d}=$ $\left(d_{k, n}\right)^{-\mu / 2} \cdot \tilde{\varphi}_{k, n}^{l / 2} \cdot \varphi_{k, n}^{k / 2} \cdot \alpha_{k, n} \cdot e^{j \theta_{k, n}}, Q_{r}=\left(\hat{d}_{k, n}\right)^{-\mu / 2}$. $\hat{\varphi}_{k, n}^{1 / 2} \cdot \ddot{\varphi}_{k, n} \cdot \tilde{\varphi}_{k, n}^{l / 2} \cdot \hat{\alpha}_{k, n} \cdot e^{j \hat{\theta}_{k, n}}$ and $\eta_{n}(t)$ is the complexvalued additive white Gaussian noise (AWGN) received by the $n$-th RAU, which has a zero mean and a double sided power spectral density $N_{0} / 2$. Assuming the phase and the path delay are known at the receiver and the receiver has a 
perfect timing synchronisation with the ME- $k^{\prime}$ i.e., $\tau_{k^{\prime}, n}=0$, the demodulated signal of ME- $k^{\prime}$ over one symbol period $T_{s}$ is given by

$$
\begin{aligned}
Z_{n} & =\operatorname{Re}\left\{\frac{1}{T_{s}} \int_{0}^{T_{s}} r_{k, n}(t) \cdot e^{-j 2 \pi f_{c} t} \cdot e^{-j \theta_{k^{\prime}, n}} d t\right\} \\
& =\operatorname{Re}\left[S_{n}+I_{n}\right]+\eta_{n}
\end{aligned}
$$

where $S_{n}$ is the desired signal component received by the $n$-th RAU on the reference floor, given by

$$
\begin{aligned}
S_{n} & =\frac{1}{T_{s}} \int_{0}^{T_{s}} D_{s} \cdot x_{k^{\prime}}(t) \cdot e^{-j \theta_{k^{\prime}, n}} \cdot e^{-j 2 \pi f_{c} t} d t \\
& =\sqrt{P_{s}} \cdot\left(d_{k^{\prime}, n}\right)^{-\mu / 2} \cdot \tilde{\varphi}_{k^{\prime}, n}^{l / 2} \cdot b_{k^{\prime}} \cdot \alpha_{k^{\prime}, n}
\end{aligned}
$$

$I_{n}$ is the total co-channel interference term received by the $n$-th RAU on the reference floor, given by

$$
I_{n}=\sum_{k=1, k \neq k^{\prime}}^{K} I_{k, n}
$$

where $I_{k, n}$ is the co-channel interference component from other floors, given by

$$
\begin{aligned}
I_{k, n}=\frac{1}{T_{s}} \int_{0}^{T_{s}} & \left(G_{d} \cdot x_{k}\left(t-\tau_{k, n}\right) \cdot e^{-j \theta_{k, n}}\right. \\
& \left.+Q_{r} \cdot x_{k}\left(t-\hat{\tau}_{k, n}\right) \cdot e^{-j \hat{\theta}_{k, n}}\right) d t
\end{aligned}
$$

Since $E\left[\alpha_{k, n}^{2}\right]=1, E\left[b_{k}\right]=0, E\left[b_{k} \cdot b_{k}^{*}\right]=1$ and $E\left[\tau_{k, n}^{2}\right]=$ $E\left[\left(T_{s}-\tau_{k, n}\right)^{2}\right]=\frac{T_{s}^{2}}{3}$, and similarly, $E\left[\hat{\tau}_{k, n}^{2}\right]=E\left[\left(T_{s}-\right.\right.$ $\left.\left.\hat{\tau}_{k, n}\right)^{2}\right]=\frac{T_{s}^{2}}{3}$, the real and imaginary parts of $I_{k, n}$ have the same variance derived as

$$
\begin{aligned}
\sigma_{I_{k, n}}^{2}=\frac{P_{s}}{3} \cdot\left[\left(d_{k, n}\right)^{-\mu} \tilde{\varphi}_{k, n}^{l} \varphi_{k, n}^{k}\right. \\
\\
\left.+\left(\hat{d}_{k, n}\right)^{-\hat{\mu}} \hat{\varphi}_{k, n} \ddot{\varphi}_{k, n}^{2} \tilde{\varphi}_{k, n}^{l}\right]
\end{aligned}
$$

According to [10], the total co-channel interference term $I_{n}$ is a zero mean Gaussian distributed random variable. Thus, the expectation of $I_{n}$ is a sum of complex Gaussian random variables easily derived as $E\left[I_{n}\right]=0$ and the variance denoted by $\sigma_{I_{n}}^{2}$ is derived as

$$
\begin{aligned}
\sigma_{I_{n}}^{2}= & E\left[I_{n} \cdot I_{n}^{*}\right] \\
= & \frac{P_{s}}{3} \sum_{k=1, k \neq k^{\prime}}^{K}\left[\left(d_{k, n}\right)^{-\mu} \tilde{\varphi}_{k, n}^{l} \varphi_{k, n}^{k}\right. \\
& \left.\quad+\left(\hat{d}_{k, n}\right)^{-\hat{\mu}} \hat{\varphi}_{k, n} \ddot{\varphi}_{k, n}^{2} \tilde{\varphi}_{k, n}^{l}\right]
\end{aligned}
$$

In (7), $\eta_{n}$ is the noise component with zero mean and a variance of $\sigma_{\eta}^{2}=N_{0} /\left(2 T_{s}\right)$, which is assumed to be equal for all RAUs.

For diversity reception, the minimum mean-square error combining (MMSEC) is employed at the receiver. Accordingly, the decision variable for detecting the desired signal is expressed as

$$
Z_{0}=\sum_{n=1}^{N} W_{n} \cdot Z_{n}
$$

where $W_{n}$ is the optimum weight vector which maximizes the SINR. $W_{n}$ can be expressed as

$$
W_{n}=\frac{\alpha_{k^{\prime} n}}{\rho_{n}}\left(d_{k^{\prime}, n}\right)^{-\mu / 2} \tilde{\varphi}_{k^{\prime}, n}^{l / 2}
$$

where $\rho_{n}$ is the variance of the interfering signal plus background noise at the $n$-th RAU on the reference floor, written as

$$
\rho_{n}=E\left[I_{n} \cdot I_{n}^{*}\right]+\sigma_{\eta}^{2}=\sigma_{I_{n}}^{2}+\sigma_{\eta}^{2}
$$

Correspondingly, the instantaneous SINR is written as

$$
\begin{aligned}
\gamma & =\frac{\left(\sum_{n=1}^{N} W_{n} S_{n}\right)^{2}}{\sum_{n=1}^{N} W_{n}^{2} \sigma_{I_{n}}^{2}+\sum_{n=1}^{N} W_{n}^{2} \sigma_{\eta}^{2}} \\
& =\sum_{n=1}^{N} \frac{P_{s}\left(d_{k^{\prime}, n}\right)^{-\mu} \tilde{\varphi}_{k^{\prime}, n}^{l}}{\rho_{n}} \alpha_{k^{\prime}, n}^{2}
\end{aligned}
$$

The pdf of the instantaneous SINR, $\gamma$, in (16), for arbitrary values of the Nakagami fading parameters is then obtained as [27]

$$
P_{\gamma}(\gamma)=\frac{1}{\pi} \int_{0}^{\infty} \frac{\cos \left[\sum_{n=1}^{N} m_{n} \tan ^{-1}\left(\frac{t}{\beta_{n}}\right)-t \gamma\right]}{\prod_{n=1}^{N}\left(1+\left(\frac{t}{\beta_{n}}\right)^{2}\right)^{m_{n} / 2}} d t
$$

where $\beta_{n}=m_{n} / \bar{\gamma}_{n} \cdot \bar{\gamma}_{n}$ is the average SINR per RAU given by

$$
\bar{\gamma}_{n}=\frac{\frac{2 E_{s}}{N_{0}} \cdot\left(d_{k^{\prime}, n}\right)^{-\mu} \tilde{\varphi}_{k^{\prime}, n}^{l}}{\frac{2}{3} \cdot \frac{E_{s}}{N_{0}}\left(\sum_{k=1, k \neq k^{\prime}}^{K} \tilde{I}\right)+1} \cdot \Omega_{n}
$$

where $\frac{E_{s}}{N_{0}}$ is the transmit symbol energy-to-noise density ratio at the ME transmitter location and $E_{s}$ is expressed as

$$
E_{s}=P_{s} \cdot T_{s}
$$

\section{Performance Analysis}

In order to maximize spectrum efficiency, the adaptive modulation is employed to combat the effect of fading. This technique allows the indoor DAS to use the highest order modulation to send more bit per symbol depending on channel conditions. Under a certain modulation order $M_{\hat{j}}=2^{\hat{j}}$ for QAM, the relationship between bit error rate $\hat{P}_{e}(\gamma)$ and SINR $\gamma$ can be expressed as [28]

$$
\hat{P}_{e}(\gamma)=\frac{1}{5} \exp \left[\frac{-3 \gamma}{2\left(2^{j}-1\right)}\right]
$$

where $\hat{j}$ is the number of bits per symbol. Given a target instantaneous BER equal to $\hat{P}_{0}$, the adaptive modulator switching thresholds $\gamma_{\hat{j}}$ for switching across the modulation orders can be solved as shown in [9]. The average spectral efficiency measured in unit of bits per second per Hertz is defined as the sum of the data rates, $\log _{2}\left(M_{\hat{j}}\right)=\hat{j}$, weighted by the probability that the $\hat{j}$-th modulation constellation is assigned to ME- $k^{\prime}$ and expressed as

$$
T_{u}=\sum_{\hat{j}=1}^{\hat{J}} \log _{2}\left(M_{\hat{j}}\right) \int_{\gamma_{\hat{j}}}^{\gamma_{\hat{j}+1}} P_{\gamma}(\gamma) d \gamma
$$




\section{Numerical Results}

In this section, the analytic formulas derived in Sections II and III are numerically evaluated for 10000 possible ME locations across a hypothetical 7-storey building. The dimension of the floor is $40 \mathrm{~m} \times 40 \mathrm{~m}$ with an inter-floor spacing $F$ of $4 \mathrm{~m}$, the MEs are evenly located at a height $v$ of $1 \mathrm{~m}$ above the floor and there are 4 RAUs on each floor. In evaluating the average value of spectral efficiency across the entire floor, pathloss exponent $\lambda$, floor penetration loss $\varphi$, wall penetration loss $\tilde{\varphi}$, reflection loss $\hat{\varphi}$ and transmission coefficient through a glass of window $\ddot{\varphi}$ are assumed to be $2.5,13 \mathrm{~dB}$, $6.5 \mathrm{~dB}, 8 \mathrm{~dB}$ and $0.13 \mathrm{~dB}$ respectively. This is consistent with measurement campaigns reported in [21]. Nakagami fading values $\left\{m_{1}, m_{2}, m_{3}, m_{4}\right\}=\{1.8,1.5,1.25,1.0\}$ and there is a distance of $10 \mathrm{~m}$ between the reference and the adjacent building. The transmit SNR $E_{s} / N_{0}$ and target BER $\hat{P}_{0}$ are set to $30 \mathrm{~dB}$ and $10^{-3} \mathrm{~dB}$ respectively.

Fig. 2 shows the average spectral efficiency for frequency reuse distances of $C=1,2,3$ floors. Note that a frequency reuse distance of 2 floors implies that the same frequencies are reused every two floors (co-channel interfering users are located on the 3rd, 5th and 7th floors). It is observed that increasing the reuse distance from one floor to three floors improves the performance significantly, especially at high SNR regions. This is due to high inter-floor isolation of cochannel floors which ensures high SINR, and high spectral efficiency across the floor. It can be seen from the figure that higher spectral efficiency values are achieved when the reuse distance is increased from one floor to two floors than when it is increased from two floors to three floors. This indicates that most of the co-channel interference emanates from users operating on close adjacent floors. An increase in the reuse distance from two floors to three floors results in a relatively small increase in spectral efficiency values, especially at low SNR regions. Further increases in reuse distance only result in little additional gains in spectral efficiency across the floor. Note that, channel noise becomes dominant when interference is weak, thus, at SNR regions below $35 \mathrm{~dB}$, spectral efficiency increases linearly. However, at SNR values more than about $40 \mathrm{~dB}$, the curves tend to be flat because cochannel interference dominates channel noise.

Fig. 3 shows the achievable spectral efficiency for scenarios with different path loss exponent values. Path loss exponent values in indoor environments are generally determined by the internal layout of the building. Lower path loss exponent values represent environments with fewer obstacles between the MEs and RAUs, while higher values represents a NLOS environment with more obstructed paths. It is observed from Fig. 3 that buildings with higher pathloss exponent achieve lower system performance. This is due to increase in the path loss of the desired ME. Although a higher path loss exponent value results in greater level of isolation from co-channel floors, thereby reducing the impact of co-channel interference, the power of the desired ME is also reduced due to increased path loss, resulting in lower spectral efficiency values. It is also observed that increasing the reuse distance from one to three floors improves the performance considerably in scenarios with lower path loss values, whereas it has little or no effect in buildings with more obstructed paths between the MEs and RAUs especially at low SNR regions. At high SNR regions, an increase in reuse distance results in a relatively small increase in spectral efficiency for buildings with higher path loss exponent values when compared with scenarios with lower path loss exponent. This is due to high transmission path loss of the desired ME which dominates the achievable spectral efficiency.

Fig. 4 shows the achievable spectral efficiency for a range of floor penetration losses with respect to frequency reuse distances of $C=1,2,3$ floors. Depending on the composition of the floors inside the building, a significant reduction in the total received signal to interference power is due to floor penetration losses. If the floors of the building are made of corrugated steel panels of about $20 \mathrm{~dB}$ penetration loss for example, an evaluation of the potential spectral efficiency can be made. It is observed from the figure that reuse distance and the achievable spectral efficiency depend strongly on the composition of the floor. The spectral efficiency increases with increasing penetration loss values. This is due to attenuation of interfering signals as penetration loss increases. Buildings with low penetration loss less than $4 \mathrm{~dB}$ may not tolerate a reuse distance of one floor due to low interfloor isolation which results in low SINR, and low spectral efficiency values. Results also indicate that co-channel users located more than two floors away contribute relatively small total interference power. Thus, higher spectral efficiency is achieved when reuse distance increases from one floor to two floors than when increased from two to three floors.

\section{CONCLUSION}

This paper has examined the performance of indoor DAS employing frequency reuse. The performance of the system is analytically quantified in terms of spectral efficiency for a 7storey building by considering an indoor propagation channel model derived from multi-floor, in-building measurement data. The results indicate that the performance of the indoor DAS is highly sensitive to propagation parameters of the building. It has been shown that beyond immediately adjacent co-channel floors, an increase in reuse distance does not always result in substantial increase in performance. Results indicate that an entire building can reuse a small allocation of spectrum across the floors.

\section{REFERENCES}

[1] T. S. Rappaport et al., "Millimeter wave mobile communications for 5G cellular: It will work!", IEEE Access, vol. 1, pp. 335349, May 2013.

[2] H. Claussen, L. T. W. Ho, and L. G Samuel, "An overview of the femtocell concept”, Bell Labs Tech. J., 13:1 (2008), 221 - 245.

[3] R. W. Heath, N. Gonzlez-Prelcic, S. Rangan, W. Roh, and A. M. Sayeed, "An overview of signal processing techniques for millimeter wave mimo systems," IEEE Journal of Selected Topics in Signal Processing, vol. 10, no. 3, pp. 436-453, April 2016.

[4] D. Nguyen, L. Tran, P. Pirinen and M. Latva-aho, "On the Spectral Efficiency of Full-Duplex Small Cell Wireless Systems," IEEE Transactions on Wireless Communications, vol. 13, no. 9, pp. 4896-4910, Sept. 2014.

[5] H. Wei, D. Wang, H. Zhu, J. Wang, S. Sun, and X. H. You, "Mutual coupling calibration for multiuser massive MIMO systems," IEEE Transactions on Wireless Communications, vol. 15, pp. 606-619, January 2016. 


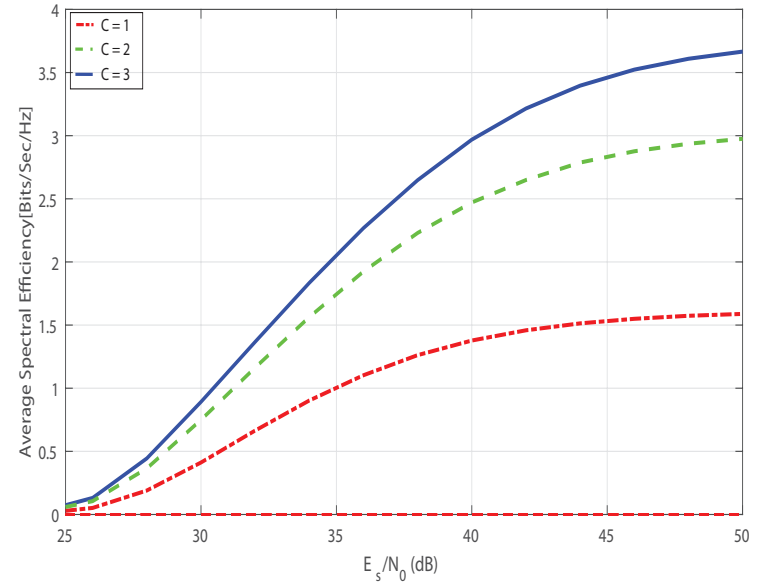

Fig. 2. Spectral efficiency for different reuse distances

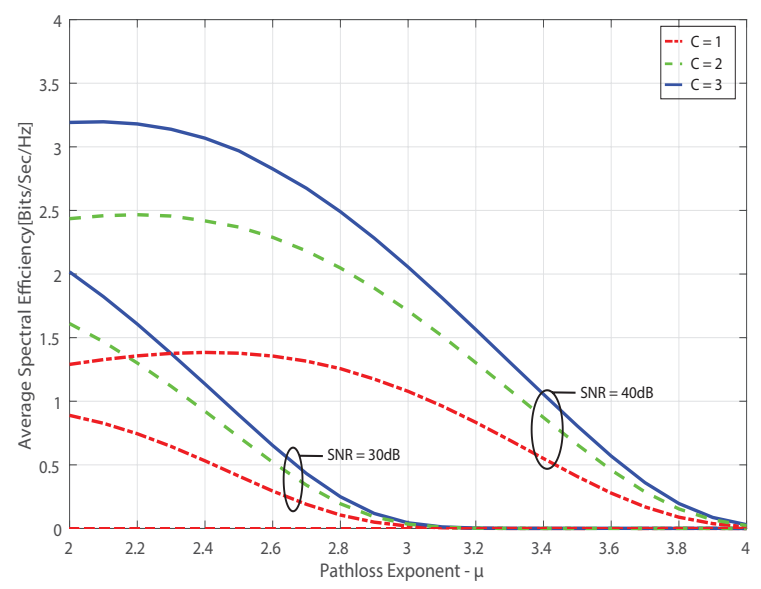

Fig. 3. Effect of path loss exponent on achievable spectral efficiency for different reuse distances

[6] Y. Xin, D. Wang, J. Li, H. Zhu, J. Wang, and X. H. You, "Area spectral efficiency and area energy efficiency of massive MIMO cellular systems," IEEE Transactions on Vehicular Technology, vol. 65, pp. 3243-3254, no. 5, May 2016.

[7] J. Wang, H. Zhu, L. Dai, N. J. Gomes, and J. Wang, "Low-Complexity Beam Allocation for Switched-Beam Based Multiuser Massive MIMO Systems", IEEE Transactions on Wireless Communications, vol. 15, pp. 8236-8248, no. 12, December 2016.

[8] H. Ngo, A. Ashikhmin, H. Yang, E. Larsson and T. Marzetta, "Cell-Free Massive MIMO Versus Small Cells," IEEE Transactions on Wireless Communications, vol. 16, no. 3, pp. 1834-1850, Jan. 2017.

[9] T. Alade, H. Zhu, and J. Wang, "Uplink spectral efficiency analysis of in-building distributed antenna systems," IEEE Transactions on Wireless Communications, vol. 14, no. 7, pp. 4063-4074, July 2015.

[10] Huiling Zhu, "Performance Comparison between Distributed Antenna and Microcellular Systems," IEEE Journal on Selected Areas in Communications, vol. 29, no. 6, pp. 1151-1163, June 2011.

[11] J. Wang, H. Zhu, and N. Gomes, "Distributed antenna systems for mobile communications in high speed trains," IEEE Journal on Selected Areas in Communications, vol. 30, pp. 675-683, May 2012.

[12] H. Zhu, S. Karachontzitis and D. Toumpakaris, "Low-complexity Resource Allocation and Its Application to Distributed Antenna Systems, IEEE Wireless Communications Magazine, vol. 17, no. 3, pp. 44-50, June, 2010

[13] H. Zhu and J. Wang, "Radio resource allocation in multiuser distributed antenna systems," IEEE Journal on Selected Areas in Communications,

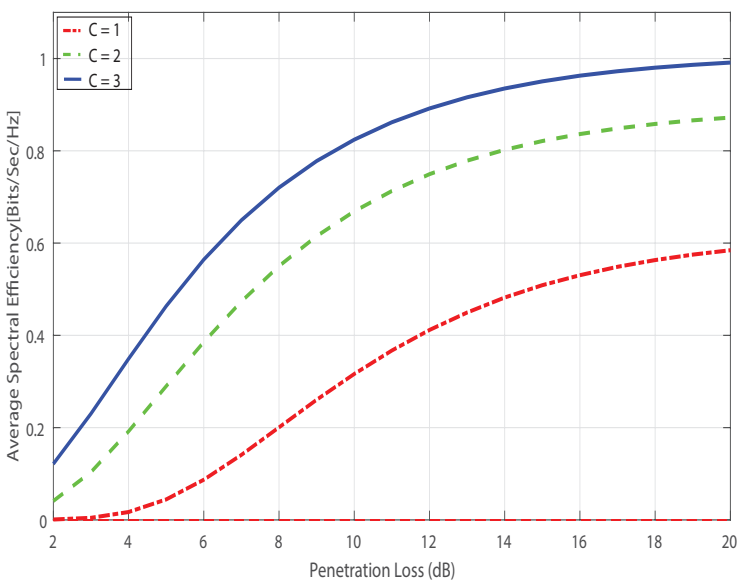

Fig. 4. Effect of penetration loss on achievable spectral efficiency for different reuse distances

vol. 31, no. 10, pp. 2058-2066, October 2013.

[14] H. Zhu, "On frequency reuse in cooperative distributed antenna systems," IEEE Communications Magazine, vol. 50, pages 85-89, Issue 4 April 2012.

[15] C. Pan, H. Zhu, N. J. Gomes, and J. Wang, "Joint Precoding and RRH selection for User-centric Green MIMO C-RAN," IEEE Transactions on Wireless Communications, vol. 16, pp. 2891-2906, no. 5, May 2017.

[16] W. Ni, R. Liu, I. Collings and X. Wang,"Indoor cooperative small cells over ethernet," IEEE Communications Magazine, vol. 51, no. 9 , pp. 100-107, Sept. 2013

[17] W. M. Thiel and K. Sarabandi,"A Hybrid Method for Indoor Wave Propagation Modeling," IEEE Transactions on Antennas and Propagation, vol. 56, no. 8, pp. 2703-2709, Sept. 2008.

[18] M. A. Panjwani, A. L. Abbott, and T. S. Rappaport, "Interactive computation of coverage regions for wireless communication in multifloored indoor environments," IEEE J. Select. Areas Commun., vol. 14, pp.420430, Apr. 1996.

[19] W. Honcharenko, H. L. Bertoni and J. Dailing,"Mechanism governing propagation between different floors in buildings"; IEEE Trans. Antennas Propagation, vol. 41. no. 6, pp 787-790 June 1993.

[20] Andrew C. M. Austin, Michael J. Neve, Gerard B. Rowe and Ryan J. Pirkl, "Modelling the effects of nearby buildings on inter-floor radio wave propagation"; IEEE Trans. Antennas Propagation., vol. 57. no. 7, pp. 2155-2161 July 2009.

[21] Recom. ITU-R P.1238-6,"Propagation data and prediction methods for the planning of indoor radiocommunication systems and radio local area networks in the frequency range $900 \mathrm{MHz}$ to $100 \mathrm{GHz}$ "; 2017.

[22] H. Jin and V. Leung, "Performance Analysis of Full-Duplex Relaying Employing Fiber-Connected Distributed Antennas," IEEE Transactions on Vehicular Technology, vol. 63, no. 1, pp. 146-160, Jan. 2014.

[23] H. Zhu and J. Wang, "Chunk-based resource allocation in OFDMA systems - Part I: chunk allocation," IEEE Transactions on Communications, vol. 57, no. 9, pp. 2734-2744, Sept. 2009.

[24] H. Zhu and J. Wang, "Chunk-based resource allocation in OFDMA systems - Part II: joint chunk, power and bit allocation," IEEE Transactions on Communications, vol. 60, no. 2, pp. 499-509, Feb. 2012.

[25] H. Zhu, "Radio resource allocation for OFDMA systems in high speed environments," IEEE Journal on Selected Areas in Communications, vol. 30, pp. 748-759, May 2012.

[26] H. Zhu and J. Wang, "Performance analysis of chunk-based resource allocation in multi-cell OFDMA systems," IEEE Journal on Selected Areas in Communications, vol. 32, no. 2, pp. 367-375, February 2014

[27] G. Efthymoglou and V. Aalo, "Performance of RAKE receivers in Nakagami fading channel with arbitrary fading parameters", IEEE Electronic Letters, Vol.31, No.18, pp. 1610-1612, Aug. 1995.

[28] M. S. Alouini and A. J. Goldsmith, "Adaptive modulation over Nakagami fading channels," Wireless Pers. Commun., vol. 13, pp. 119 143, May 2000. 